\title{
Les enjeux de la mémoire dans The Conspiracy and Tragedy of Byron de George Chapman (1608)
}

\author{
Gilles Bertheau \\ Christophe Hausermann (éd.)
}

\section{(2) OpenEdition \\ Journals}

\section{Édition électronique}

URL : http://journals.openedition.org/shakespeare/1919

DOI : 10.4000/shakespeare.1919

ISSN : 2271-6424

\section{Éditeur}

Société Française Shakespeare

Édition imprimée

Date de publication : 1 avril 2013

Pagination : 27-42

ISBN : 2-9521475-9-0

Référence électronique

Gilles Bertheau, "Les enjeux de la mémoire dans The Conspiracy and Tragedy of Byron de George Chapman (1608) ", Actes des congrès de la Société française Shakespeare [En ligne], 30 | 2013, mis en ligne le 03 avril 2013, consulté le 20 avril 2019. URL : http://journals.openedition.org/ shakespeare/1919; DOI : 10.4000/shakespeare.1919 


\section{Shakespeare et la mémoire}

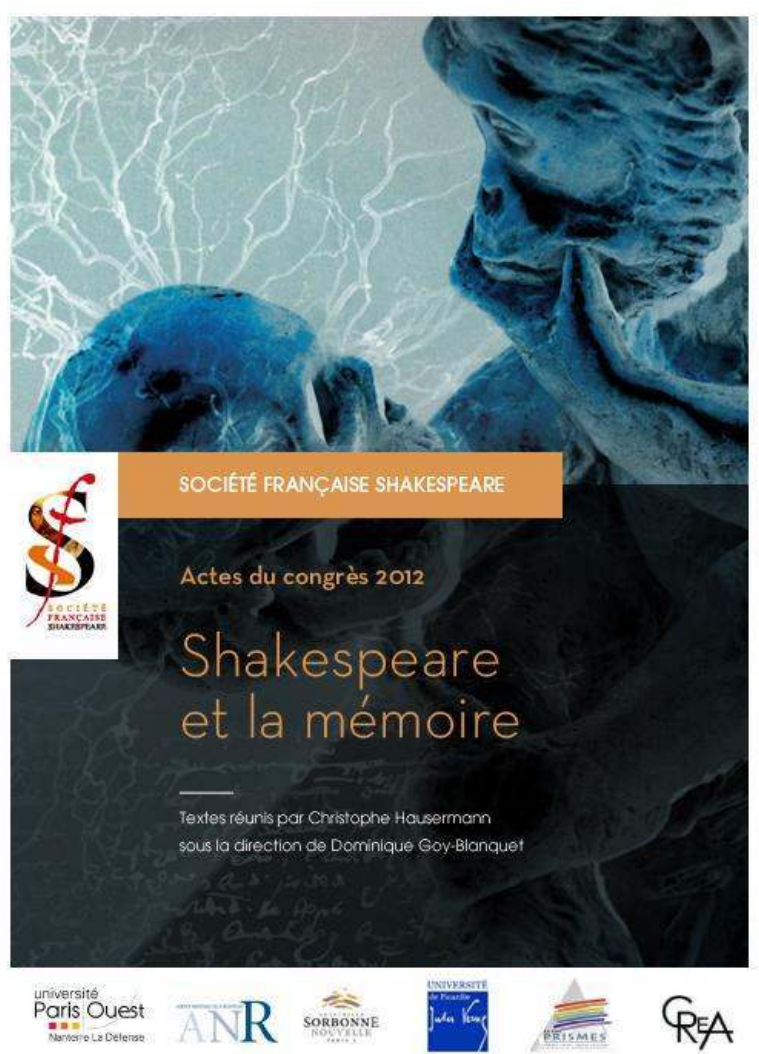

actes du Congrès

organisé par la

SOCIÉTÉ FRANÇAISE SHAKESPEARE

les 22, 23 et 24 mars 2012

textes réunis par

Christophe HAUSERMANN

sous la direction de

Dominique GoY-BLANQUET 


\section{COUVERTURE}

d'après l'affiche de Claire Colombet

conception graphique et logo

Pierre Kapitaniak

mise en page et corrections

Christophe Hausermann

(C) 2012 Société Française Shakespeare

Institut du Monde Anglophone

Université de Paris III - Sorbonne Nouvelle

5 rue de l'École de Médecine 75006 Paris

www.societefrancaiseshakespeare.org

Tous droits de traduction, de reproduction et d'adaptation réservés pour tous les pays 


\title{
LES ENJEUX DE LA MEMOIRE DANS THE CONSPIRACY AND TRAGEDY OF BYRON DE GEORGE CHAPMAN (1608)
}

\author{
Gilles BERTHEAU
}

\begin{abstract}
Écrite deux ans avant la mort d'Henri IV, The Conspiracy and Tragedy of Byron de George Chapman met en scène la lutte politique inégale entre Byron, nostalgique d'une monarchie idéalisée dont le moteur est la guerre, et Henry, roi moderne déterminé à reconstruire un royaume de paix. Le but de cet article est de montrer que l'affrontement entre les deux personnages passe le plus souvent par une bataille autour de la mémoire des événements relativement récents qui se sont produits depuis la mort d'Henri III jusqu'au début de la conspiration du duc. Manipulée par Savoy (le duc de Savoie) à des fins personnelles, cette mémoire est l'occasion pour le roi et le maréchal de Byron d'une surenchère où la vanité de l'un et de l'autre fait fi de la réalité historique. Car l'enjeu, qui est ailleurs, est avant tout d'ordre symbolique et donc politique : Henry, qui veut s'affirmer comme roi « moderne » et absolu, entend bien conserver tout le bénéfice - et le monopole - de la gloire militaire acquise depuis 1589 , et ce au détriment de son maréchal, qui pense naïvement que le roi a une dette envers lui. Devant la résistance du duc, et devant sa puérile traîtrise, le roi en viendra à effacer cette dette en éliminant physiquement - et symboliquement - le rebelle, incarnation de la mémoire des années d'avant la prise de pouvoir effective du roi. Le portrait de Henry dans le diptyque frappe par ses ressemblances avec le Henri IV historique qui, en 1598, met en place l'Édit de Nantes, dont les deux premiers articles prescrivent un oubli officiel des conflits religieux passés. Ainsi, il semble que pour le personnage et pour son modèle, l'histoire se construise sur un art de l'oubli, pour paraphraser Paul Ricœur.
\end{abstract}

Two years before the death of Henri IV of France, George Chapman, in The Conspiracy and Tragedy of Byron, staged the unequal political fight between the duke of Byron, still living in the dream of an idealised monarchy whose main function is to wage war, and Henry, a modern king who is determined to restore his kingdom to peace. The aim of this article is to show that the confrontation between the two characters mostly happens as a battle for the memory of the relatively recent events that took place between the death of Henri III and the beginning of the duke's conspiracy. Shamelessly manipulated by the duke of Savoy in his own interest, this memory helps to reveal Byron's and Henry's vanity, the duke and the king having no care for the reality of history. Indeed, the problem is first and foremost symbolic, and therefore political: Henry, who wants to assert himself as a "modern" and absolute king, will not let anyone - even Byron - deprive him of the sole benefit of the military glory he has harvested since 1589. This is done to the detriment of Byron, who naively thinks that the king owes him. As the duke will not yield, and perseveres in his treason, the king removes the debt by physically removing the rebel who too well embodied the memory of the years before the king came into his own. This presentation of Henry in the double play is strikingly reminiscent of the historic Henri IV who, in 1598, implemented the Edict of Nantes, the first two articles of which command to forget the past civil wars. It therefore seems that, for both the character and his model, history has more to do with oblivion than with memory.

$\mathrm{P}$ arue en 1608, The Conspiracy and Tragedy of Byron est plus une pièce d'actualité qu'une pièce historique ${ }^{1}$ : en effet, les événements que George Chapman met en scène ont eu lieu neuf ou dix ans auparavant, le plus ancien étant la signature à Vervins du traité de paix avec l'Espagne, en 1598, qui ouvre une nouvelle séquence

${ }^{1}$ Voir Christine Sukič, Le Héros inachevé : éthique et esthétique dans les tragédies de George Chapman (1559?-1634), Berne, Peter Lang, 2005, p. 174. 
dans le règne d'Henri IV. Dans le diptyque, trois personnages principaux (Savoy, Byron et Henry) évoquent des faits antérieurs qui se sont déroulés entre la mort d'Henri III en 1589 et la prise d'Amiens en 1597. Il s'agit des batailles victorieuses menées par le roi et ses alliés dont Byron - contre les forces de la Ligue menées par le duc de Mayenne. Pour eux aussi, le passé en question est donc très récent et son souvenir est encore extrêmement vif dans leur mémoire et celle de tous les personnages.

Pourtant, malgré cette proximité temporelle, Chapman nous donne à entendre des versions fort différentes selon les personnages, dont aucun ne semble réellement animé par un souci d'exactitude. L'enjeu n'est pas, en effet, de faire le récit du réel, comme un historien, mais de défendre une position stratégique en construisant un récit personnel. D'emblée, le dramaturge nous met en garde en mettant en scène la manipulation de la mémoire par Savoy, à des fins politiques. Cette première évocation du passé provoque un conflit mémoriel entre Byron et Henry, dont l'issue est fatale au premier. Tandis que le maréchal ne cesse d'exalter ses faits d'armes, et pense prouver ainsi son infaillible loyauté envers Henry, ce dernier, en livrant bataille pour sa propre gloire (une bataille rhétorique cette fois), cherche et réussit à imposer une version du passé qui le libère de sa dette envers le maréchal et assoit définitivement son pouvoir absolu. Dans un système politique où la gloire ne peut plus se partager, Henry choisit la postérité pour lui-même et l'oubli promis aux traîtres pour Byron. C'est pourquoi il est essentiel pour les deux personnages de contrôler le récit de leur passé, et donc leur réputation, pour les générations à venir.

La rhétorique du souvenir, ou les éléments constitutifs du discours de la mémoire

Il s'agit ici de dresser ce que Paul Ricœur appelle une "phénoménologie » de la mémoire ${ }^{2}$. Celle-ci est liée, dans la pièce, à trois éléments.

Le premier se rattache à une géographie du souvenir, qui se confond avec la géographie des batailles livrées et gagnées par Henry, avec Byron à ses côtés, entre 1589 et 1597 . Ces lieux sont d'abord rappelés par le duc de Savoie, au début de The Conspiracy of Byron.

\footnotetext{
${ }^{2}$ Paul Ricœur, La Mémoire, l'histoire, l'oubli, Paris, Arléa, 200o, p. 5.
} 
C'est ainsi qu'on l'entend énumérer les noms des villes de Dreux, de Beaune, de Dijon et de Fontaine Française $\left(C B^{3}\right.$, II.ii.112, 137, 140 et 144), batailles gagnées en 1590 et 1595 .

L'évocation de ce passé récent par le personnage du duc de Savoie dès le début de la pièce permet à Chapman de faire de la mémoire une question problématique. En effet, puisque le passé a eu lieu, le témoin d'un événement peut dire " J’y étais », comme nous le rappelle Paul Ricœur ${ }^{4}$. Or justement, Savoy n'y était pas ; il ne peut dire « J'y étais 5 » et ne peut donc pas associer le temps et l'espace. Aussi en est-il réduit à déclarer, à propos de Byron, «Il y était ». En outre, ce qu'il dit n'est pas le souvenir d'une action passée, mais le souvenir d'un récit de cette action, entièrement fondé sur des rumeurs : " I have heard » $(C B$, II.ii.109) et " I only tell your highness what I heard » $(C B$, II.ii.200). En quelque sorte, c'est une mémoire usurpée, une fausse mémoire, la mémoire d'un passé qui n'est pas celui du personnage ${ }^{6}$. Et pourtant, dans cette réédition rhétorique des batailles de Dreux ( $C B$, II.ii.112-132) et de Fontaine Française ( $C B$, II.ii.144-197), qui occupent le centre de son intervention, Savoy raconte l'événement comme s'il avait été présent, avec force détails tendant à accréditer ce qui pourrait presque passer pour un témoignage. La manipulation à l'œuvre dans cette scène est donc double : il s'agit pour le duc de Savoie de créer un conflit entre Henry et Byron, et pour Chapman de nous mettre en garde, au début de ce diptyque, contre les usages frauduleux de la mémoire.

Le but affiché de Savoy est atteint puisqu'il force le roi, non pas à dire : " J'y étais », mais à demander : "Was not I there? » $(C B$, II.ii.209), question rhétorique qui le place en position défensive et semble presque jeter un doute quant à sa présence sur les champs de batailles évoqués.

\footnotetext{
3 Nous utiliserons les abréviations $C B$ pour The Conspiracy of Byron et $T B$ pour The Tragedy of Byron.

${ }^{4}$ Ibid., p. 183: « La déclaration explicite du témoin [...] le dit bien : 'J’y étais.' L'imparfait grammatical marque le temps, tandis que l'adverbe marque l'espace. »

5 Il marque aussi par là que le passé en question a déjà cessé d'appartenir à un homme en particulier, Byron ou Henry, et qu'il fait déjà partie en quelque sorte de la mémoire collective, celle de l'histoire de France.

${ }^{6}$ La mémoire de Savoy est une fausse mémoire qui ne correspond pas à la définition qu'en donne Paul Ricœur : « On l'a dit avec Aristote, on le redit plus fortement avec Augustin, la mémoire est du passé et ce passé est celui de mes impressions ; en ce sens, ce passé est mon passé, » op. cit., p. 116.
} 
Deux autres éléments servent à construire le récit : les chiffres et les blessures. Parmi les détails donnés par Savoy, les chiffres sont particulièrement frappants. Ils lui permettent de rendre son récit crédible et d'exalter le mérite de Byron au détriment du roi. C'est ainsi que Byron, dit-il, eut à se battre contre 4000 cavaliers et 12000 fantassins à Dreux $(C B$, II.ii.114-15) et contre 1200 cavaliers à Fontaine Française ( $C B$, II.ii.162). Il raconte comment le maréchal dut lutter contre cinq compagnies de cavaliers avec seulement vingt hommes ( $C B$, II.ii.166). Savoy termine son récit de la bataille de Fontaine Française en déclarant à propos de Byron : " And only with a hundred gentlemen / He won the place, from fifteen hundred horse » $(C B$, II.ii.196-97) et conclut en posant cette question : «But is it not most true that 'twixt you both / So few achieved the conquest of so many? » ( $C B$, II.ii.206-207), plaçant ainsi Henry et son maréchal sur un pied d'égalité. Mais ces chiffres, encore réalistes à ce moment de l'action, se trouvent multipliés de manière extravagante par Byron quand Vitry vient l'arrêter, à la fin de The Tragedy of Byron. Le duc lui demande ainsi : "Will you smother here / His life that can command and save in field / A hundred thousand lives? » (TB, Iv.ii.243-45). Cette inflation en annonce une autre, celle du nombre de ses blessures, le troisième élément de cette phénoménologie de la mémoire.

La blessure incarne littéralement la trace, l'empreinte laissée par le passé sur le corps du soldat qu'est Byron. Au départ, lorsque le duc de Savoie en parle, les blessures de Byron ne sont que deux : une à la tête et une autre à la poitrine $\left(C B\right.$, II.ii.168-69) ${ }^{7}$. Il mentionne aussi le sang qu'il a perdu $(C B \text {, II.ii.195 })^{8}$. Il n'y a là rien d'exceptionnel. Mais à la fin de The Conspiracy of Byron, quand Henry refuse de lui accorder le commandement de la citadelle de Bourg-en-Bresse, c'est Byron lui-même qui reprend ce thème pour marteler sa loyauté : " which of all my wounds, / Which overthrow, which battle won for you, / Breeds this suspicion?» $(C B$, v.i.122-24). Précisément, la suspicion royale ravive la blessure ouverte par l'ingratitude du roi : " this reason pours / Poison for balm into the wound you made » $(C B$, II.ii.118-19), expression qui permet à Byron de transformer le roi thaumaturge ( «balm ») en empoisonneur! Ailleurs, il accuse Henry de le blesser : «It is the king [...] that injures me » (TB, v.iii.134-35), le

\footnotetext{
7 « [...] not daunted with two wounds, / One on his head, another on his breast [...]. »

8 « every drop / Of his lost blood bought with a worthy man ».
} 
mot indiquant à la fois la blessure morale et l'injustice, conformément à l'étymologie latine du verbe « injure ».

Dès lors, le thème des blessures revient de manière obsessionnelle dans le discours du maréchal, comme preuve ultime et ineffaçable de sa loyauté, à nouveau dans The Conspiracy (v.ii.26-27), mais surtout dans The Tragedy, où elles sont un motif central du ressassement dans lequel s'enferme Byron. Il les évoque cinq fois et, sans que l'on sache d'où vient ce chiffre ${ }^{9}$, il déclare par trois fois en avoir reçu trente-cinq, la première fois dans The Tragedy (v.i.129-31) : « [he] bids us / Make ourselves drunk with the remaining blood / Of five and thirty wounds received in fight / For us and ours $[\ldots]^{10}$. »

De son côté, Vitry se contente de parler de ses « diverses blessures » ( $T B$, III.ii.6). On est donc passé de deux à trente-cinq, mouvement d'inflation qui illustre bien le processus mémoriel à l'œuvre chez Byron ${ }^{11}$. Enfin, juste avant son exécution, il ne se contente plus d'en parler, il les montre à la foule : "View, view this wounded bosom » $\left(T B\right.$, v.iv.210 ${ }^{12}$, tel Ulysse (auquel Henry l'a comparé plus tôt ${ }^{13}$ ) prétendant mériter plus qu'Ajax les armes d'Achille, comme

\footnotetext{
${ }^{9}$ Edward Grimeston mentionne deux fois ce chiffre: « Sometimes hee [Byron] saide; [...] that they should speedily drinke themselues drunke, with the bloud which remained of thirty and fiue Woundes » (p. 972) et «I haue receiued fiue and thirty wounds vpon my body to preserue it for him [Henry], and for my reward, hee takes my Head from my Shoulders ». A General Inuentorie of the History of France from the Beginning of that Monarchie, vnto the Treatie of Veruins, in the Year 1598. Written by Ihon (sic) de Serres. And Continued vnto these Times, out off (sic) the Best Authors which Haue Written of that Subiect, trad. Edward Grimeston, Londres, 1607, Henry E. Huntington Library and Art Gallery, STC (2nd ed.) / 22244, p. 985.

10 « ye all know, my lords, /This body, gash'd with five and thirty wounds, /Whose life and death you have in your award, /Holds not a vein that hath not open'd been, /And which would not open yet again /For you and yours, » (TB, v.ii.206-11) et « my courage raised me, / For the dear price of five and thirty scars » (TB, v.iii.181-82).

${ }^{11}$ Ce mouvement va de pair avec un autre changement : au début, c'est le roi qu'il prend à témoin, puis, au moment de son arrestation, c'est devant Vitry, Épernon, le ministre Janin et le Vidame de Chartres, neveu de La Fin, qu'il se plaint d'être l'« enclume » sur laquelle les « coups et les blessures » reçus devaient générer de l'honneur pour d'autres que pour lui ( $T B$, IV.ii.271-72). Le cercle des témoins - et en même temps de ceux qui, d'après Byron, lui sont redevables - s'élargit pour s'étendre à tous les sujets du roi : dans la prison par exemple ( $T B$, v.i.129-32), lors de son procès ( $T B$, v.ii.206-211) et à nouveau devant ses juges lui annonçant la sentence ( $T B$, v.iii.181-83).

${ }^{12}$ Ce faisant, Byron agit à l'exact opposé de Coriolan, qui refuse à plusieurs reprises de montrer ses blessures pour être élu consul. Ainsi déclare-t-il à Ménénius : « I cannot / Put on the gown, stand naked, and entreat them / For my wounds' sake to give their suffrage, » (Coriolanus, II.ii.135-37).

13 Parlant des Espagnols qui ont essayé de corrompre Byron, le roi dit : «they found him still / As an unmatch'd Achilles in the wars, / So a most wise Ulysses to their words, / Stopping his ears at their enchanted sounds » (TB, I.i.77-80).
} 
Ovide le rapporte: «There also are (O countrimen) about mee woundings, which / The place of them make beautifull. See heere (his hand did twich / His shirt aside) and credit not vaine words ${ }^{14}$. »

Tout dans ces éléments indique la mémoire hypertrophiée du personnage éponyme. Elle le devient d'autant plus à mesure qu'il comprend que le roi veut la reléguer au second plan, puis la nier tout à fait en l'éliminant physiquement.

Insaisissable mémoire: liimpossibilité de fixer le passé sans l'injonction de l'oubli

La manœuvre politicienne - machiavélique - du duc de Savoie dans The Conspiracy of Byron fonctionne parfaitement, puisqu'elle aiguillonne l'amour-propre des deux hommes, dont l'affrontement sur le terrain de la mémoire et de la gloire constitue un motif récurrent et un élément dramaturgique central de cette double pièce. Les temps ayant changé, les deux hommes ne sont plus compagnons d'armes, et la gloire de l'un ne peut donc exister sans la négation de celle de l'autre. On ne peut comprendre les enjeux de cette querelle si on ne garde pas à l'esprit que la pièce se déroule dans un contexte de paix.

Dès le début de The Conspiracy of Byron, Chapman prend bien soin de présenter Henry comme le roi qui vient de signer le Traité de Vervins instaurant la paix avec l'Espagne $(C B$, I.i.128, 212). Il montre aussi combien Byron s'adapte mal à cette nouvelle donne politique : " O the most base fruits of a settled peace! » se lamente-t-il (TB, Iv.i.127). Il se sait inutile : « Since Bretagne is reduced, and breathless war / Hath sheathed his sword and wrapped his ensigns up, / The king hath now no more use of my valour » (TB, I.iii.5-7). Il a même conscience d'être devenu gênant pour un roi clairement qualifié de machiavélien : "My gloss, raised from the richness of my stuff, / Had too much splendour for the owly eye / Of politic ${ }^{15}$ and thankless royalty » (TB, v.ii.181-83). Et, de fait, à mesure que la trahison du maréchal devient

14 Ovide, The XV. Bookes of P. Ouidius Naso : Entituled Metamorphosis, A Worke very Pleasant and Delectable, trad. Arthur Golding, Londres, John Danter, 1593, n.p., Early English Books Online, Université Paris III Sorbonne Nouvelle, 12 juin 2012, http://gateway.proquest.com/openurl?ctx_ver=Z39.88-

2003\&res_id=xri:eebo\&rft_id=xri:eebo:image:10748:167

15 Ces paroles font écho à celles de Savoy qui appelle Henry « the politic King » ( $C B$, I.i.86), l'adjectif ayant, dans les deux cas, l'acception péjorative de "Scheming, crafty, cunning » donnée par l'OED (« politic », A.I.2.c.). 
patente, Henry s'efforce de réduire au silence le souvenir même du bruit des armes et des batailles auquel Byron veut sans cesse le ramener, alors que le roi entend inaugurer une nouvelle page de son règne, comme il le dit au personnage de La Fin $(C B$, I.i.118-24 et $T B$, III.ii.31-55).

La bataille pour la mémoire commence par l'intermédiaire de Savoy, dont le récit outrancier des exploits de Byron oblige le roi à réagir en disant, au sujet de la bataille de Dreux : « The heart but now came on $[\ldots]$ / Which, if I would be glorious, I could say / I first encountered » $(C B$, I..ii.133, 135-36). Si « glorious » a bien le sens de vaniteux, vainglorious, il reste néanmoins indissociable de cette notion de gloire, qu'Henry revendique, tout en s'en dissociant au moyen d'une prétérition. Il faut toujours garder à l'esprit que l'enjeu de ces joutes mémorielles est, in fine, d'assujettir l'autre à la dette morale et politique que représentent ses faits d'armes. Or, quelle plus grande dette que celle d'avoir sauvé la vie de l'autre ? C'est ce qu'Henry affirme devant ses conseillers: "He hath deserved my love with worthy service, / Yet can he not deny but I have thrice / Saved him from death » (TB, Iv.ii.9-11). Il donne comme exemple la bataille de Fontaine Française où il dut jouer le rôle du maréchal à la place de Byron :

\section{I drew him off the foe}

At Fontaine Françoise, where he was engaged,

So wounded, and so much amazed with blows,

That, as I play'd the soldier in his rescue,

I was enforced to play the marshal,

To order the retreat, because he said

He was not fit to do it, nor to serve me.

(TB, IV.ii.11-17)

Byron aussi se vante d'avoir sauvé la vie au roi, en expliquant à ses juges qu'il le dissuada de se rendre au Fort Sainte-Catherine, où il risquait de se faire tuer (TB, v.ii.89-95).

C'est pour la postérité que se battent Henry et Byron : chacun se soucie de l'opinion que les autres auront de lui après sa mort. À cet égard, l'exemple le plus extraordinaire est celui de la statue dont Byron rêve pour célébrer sa gloire. D'abord en bronze, il la veut ensuite taillée à même la montagne Oros pour fixer son souvenir grandiose dans le temps, infini, et dans un espace quasi-mythique (oros veut dire montagne en grec et ne renvoie donc à aucun lieu particulier). Elle le 
représenterait avec dans la main gauche la ville d'Amiens « où il servit si mémorablement », dit-il ( $C B$, III.ii.169). Cet auto-panégyrique de trente-huit vers - où le personnage parle de lui à la troisième personne $^{16}$ - est bien sûr le signe d'une vanité monstrueuse (on sait combien Byron est sensible à la flatterie) ${ }^{17}$, mais il indique aussi que le seul monument que Byron est capable d'élever à sa gloire est rhétorique. Il a bien conscience que seul l'écrit permet de transformer le passé en histoire et lui assurerait la postérité tant recherchée. Ainsi proclame-t-il à Henry :

But I will be mine own king: I will see

That all your chronicles be filled with me,

That none but I and my renownèd sire

Be said to win the memorable fields

Of Arques and Dieppe, and none but we of all

Kept you from dying there in an hospital:

None but myself that won the day at Dreux ${ }^{18}$, $\cdots$

$(T B$, v.i.137 sqq.)

Il n'est pas étonnant que le passage où Byron revendique l'autonomie de l'homme absolu coïncide avec celui où il veut à la fois confisquer toute la gloire militaire à son unique profit et faire en sorte que les « chroniques » lui rendent la justice post-mortem que le roi lui refuse. De même il supplie ses juges de garder de lui une bonne opinion :

Thanks to your lordship, and let me pray too

That you will hold good censure of my life

By the clear witness of my soul in death

That I never pass'd act 'gainst the King,

Which, if my faith had let me undertake,

He had been three years since amongst the dead.

(TB, v.iv.132-35).

On notera que cette opinion est en particulier fondée sur la piété revendiquée par Byron dans la pièce. Le contrôle du souvenir - proche

\footnotetext{
${ }^{16} \mathrm{CB}$, III.ii.166 et 177 .

${ }^{17}$ Ibid., II.i.59-69.

18 Arques et Dieppe furent prises par Henri IV en 1589, Dreux fut reprise aux Ligueurs en 1590, Fontaine Française, située près de Dijon, fut une bataille gagnée le 5 juin 1595 , Amiens reconquise le 21 septembre 1597, Douais, Béthune, Saint-Paul, Bapaume et Courcelles furent quant à elles gagnées en 1596-97. (Janine Garrisson, Henry IV, p. 151-63; Roland Mousnier, L'Assassinat d'Henri IV..., p. 105 et Georges Martin, Histoire et généalogie des maisons de Gontaut Biron et d'Hautefort, Lyon, à compte d'auteur, p. 30).
} 
du contrôle de la vérité - apparaît clairement quand Henry fait envoyer des dépêches aux rois et potentats d'Europe pour leur donner sa version - «the truth of this conspiracy » (v. 74) - de l'affaire Byron (TB, v.i.71-74).

Les pensées d'Henry vont dans la même direction, comme en témoigne le moment où le Dauphin apparaît. Dans ce passage, qui met en scène la dynastie Bourbon, le roi se projette au-delà de sa propre mort pour anticiper leur double postérité :

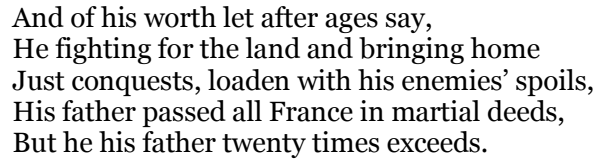

$$
\text { (TB, I.i.145-49) }
$$

Ces vers qui closent la scène prémunissent a priori le roi contre l'oubli dans la mesure où ils contiennent une référence au caractère héréditaire de la monarchie et donc, indirectement, au corps politique du souverain. Avec la présence physique de l'héritier d'Henri IV, la notion de postérité acquiert le sens qui lui manquait jusqu'à présent : par la succession, c'est non seulement le pouvoir qui se transmet, mais aussi le souvenir.

Naturellement, il n'en va pas de même pour Byron, qui n'a pas d'enfant et guère de famille. Il ne mentionne d'ailleurs que ses ancêtres, avec lesquels il veut être enterré, à Biron, berceau de la famille en Dordogne ${ }^{19}$. En outre, il se plaint amèrement d'être abandonné par les siens (ses beaux-frères et son intendant sont partis en province ${ }^{20}$ ) à cause de l'opprobre que jettera sur sa réputation sa mort ignominieuse («my opprobrious death, » $T B$, v.iv.237). De ce point de vue non plus, la lutte n'est pas égale.

Pour Henry, il s'agit non seulement de minimiser les exploits militaires de Byron et de contrôler la mémoire, mais aussi de rappeler qu'il les a richement récompensés et pense donc s'être acquitté de sa dette, comme François I ${ }^{\text {er }}$ avec Chabot $^{21}$. Mais, visiblement, cela ne

\footnotetext{
19 « [To D’Escures] Good sir, I pray, / Go after and beseech the Chancellor / That he will let my body be interred / Amongst my predecessors at Byron, » TB, v.iv.139-42.

20 «O all the world forsakes me! » TB, v.iv.62.

21 « Of all that ever shar'd in my free graces, / You Philip Chabot a meane Gentleman / Have not I rais'd you to a supremest Lord, / And given you greater dignities than any? » demande le roi à Chabot quand il voit que celui-ci résiste à sa volonté (The Tragedy of
} 
suffit pas. C'est ainsi qu'il retrace, au tout début de The Tragedy of Byron (I.i.5-19), la brillante carrière de Charles de Gontaut-Biron, quitte à tricher avec la vérité, quand il déclare que Byron a été fait colonel des Suisses à 14 ans (TB, I.i.7-8), alors qu'il avait 21 ans, en 1583, quand Henri IV l'a nommé à ce poste. En outre, ce rappel lui vaut, de la part de Byron, une remarque lucide et désabusée : « it twas not given / For any love, but fear and force of shame, » à quoi Henry répond, sans démentir : "Yet 'twas your honour » $(C B$, v.i.104-106).

Lors du dernier face-à-face entre le roi et le maréchal, Henry prononce un discours (qui n'est pas tiré de Grimeston) dans lequel il tient à faire prévaloir sa version des événements de ces années 15891597 :

Thou end the war

And settle peace in France? What war hath raged

Into whose fury I have not exposed

My person, which is as free a spirit as thine?

Thy worthy father and thyself combined

And armed in all the merits of your valours,

Never were bristled with so many battles,

Nor on the foe have broke such woods of lances

As grew upon my thigh, and I have marshalled -

I am ashamed to brag thus [...].

(TB, IV.ii.253-63)

Après avoir minimisé le rôle de Byron dans ses victoires, en expliquant que le mérite en revenait à la Fortune ( $C B$, II.ii.91), le roi, en quelques vers, sans s'abaisser à entrer dans le moindre détail, s'affranchit définitivement de sa dette en rétablissant l'ordre symbolique des choses. En quelque sorte, sa parole (quasi-performative ici) transforme le passé en histoire, Henry devient l'auctor (c'est-à-dire, au sens premier du terme latin, le garant) de sa propre histoire, confondue avec celle de la France. Face à cela, Byron n'est pas de taille à lutter ${ }^{22}$. Et pourtant, il ne désarme pas jusqu'à la fin de The Tragedy of Byron.

Chabot Admiral of France, éd. G. Blakemore Evans dans The Plays of George Chapman: The Tragedies with Sir Gyles Goosecappe, A Critical Edition, Cambridge, D. S. Brewer, 1987, II.iii.62-65).

${ }^{22}$ Les paroles du roi à ce moment du diptyque font oublier les nuances apportées par la reine Elizabeth I à l'acte IV de The Conspiracy of Byron quant à la bravoure d'Henry. 


\section{La mémoire et l'oubli comme enjeux politiques}

Comme on l'a vu, la mémoire est un objet de concurrence entre Byron et Henry. Au-delà de la gloire et de la postérité, c'est une lutte à mort qui se joue, entre un soldat dont l'identité se confond avec son passé héroïque et un roi qui doit vouer à l'oubli le souvenir de son maréchal, quitte à l'éliminer physiquement par des moyens légaux discutables, pour asseoir un absolutisme au sein duquel nulle concurrence d'autorité n'est plus envisageable ${ }^{23}$.

Le monde auquel croit Byron a, en réalité, disparu depuis longtemps : un monde où des valeurs chevaleresques gouvernaient les relations entre suzerain et vassaux, où les seconds étaient indispensables au premier par le service armé qu'ils lui rendaient. C'est pourquoi ce guerrier veut refaire le monde : "We must reform and have a new creation / Of state and government, and on our chaos / Will I sit brooding up another world ( $T B$, I.ii.29-31). Sa comparaison obsolète avec une forteresse qu'il faudrait assiéger pour la vaincre ${ }^{24}$ est pleine d'ironie puisque c'est par la ruse machiavélienne du roi (qui emploie le dénommé La Fin pour trahir Byron) qu'il sera attiré à la cour $(T B$, III.i), ce qui le conduira à sa perte. La difficulté qu'il éprouve à se séparer de son épée, qu'il compare à son "âme » (TB, IV.ii.28082), prouve combien toute son identité se rattache à son passé militaire et donc au souvenir qu'il veut que le roi en ait.

De son côté, le Henry de Chapman est pleinement conscient d'être l'artisan d'un monde nouveau dans lequel un " roi moderne 25 » ne peut être lié par aucune dette s'il entend être un roi absolu. C'est non seulement des lois qu'il doit être délié (selon la fameuse maxime romaine "princeps legibus solutus est») mais aussi des souvenirs, du moins des souvenirs qui portent ombrage à sa gloire. Leur sélection, comme il a été montré plus haut, va de pair avec la sélection des pièces à conviction qui doivent servir au procès de Byron. Henry choisit celles

\footnotetext{
23 Byron finit par comprendre qu’il est éliminé pour des raisons politiques, $T B$, v.iv.195-97. 24 Après avoir refusé de rentrer à la cour comme D’Escures le lui demandait de la part du roi, Byron déclare à D'Auvergne : «Blows, batteries, breaches, showers of steel and blood, / Must be his downright messengers for me, / And not the muzzling breath of policy. / He, he himself, made passage to his crown / Through no more armies, battles, massacres, / Than I will ask him to arrive at me [...], » (TB, III.i.130-35).

25 TB, v.iii.6o-61.
} 
où seul son nom apparait, accordant un oubli, bénéfique celui-là, à ses complices :

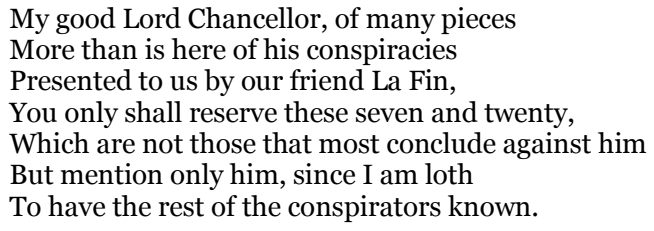

Avec le procès, la condamnation et l'exécution de Byron, Henry parvient à faire taire l'homme qui sans cesse lui rappelait, de manière outrancière certes, qu'il devait sa position actuelle à une victoire collective. Officiellement devenu traître, Byron est menacé de subir le sort de ceux qui l'ont précédé dans cette voie de perdition. Ainsi, à Henry qui déclare : «I am not yet so old but I may spring, / And then I hope all trait'rous hopes will fade », Savoy répond : "Else may their whole estates fly, rooted up / To ignominy and oblivion » $(C B$, I.i.202205). Le duc d'Aumale en fait d'ailleurs la cruelle expérience, comme il le dit à Bellièvre : «it offends me much to have my name [...] / And my whole memory with such despite / Marked and begun to be so rooted out » $(C B$, I.ii.64, 66-67). Les spectateurs, qui ont déjà entendu le prologue,

He bursts in growing great; and rising, sinks:

Which now behold in our Conspirator,

And see in his revolt how honour's flood

Ebbs into air, when men are great, not good.

sont donc avertis que le danger qui va menacer Byron n'est pas seulement la mort, mais la mort de son nom, chose bien pire que la mort, comme le rappelle Monsieur à Henry dans Bussy D'Ambois : « my friend only sau'd his fames deare life, / Which is aboue life [...] » (II.i.174-75) ${ }^{26}$.

Le discours de Byron sur l'échafaud montre qu'il est pleinement conscient de l'anéantissement de son être par l'oubli (véritable damnatio memoriae), puisqu'il déclare: «Never more/ Shall any

${ }^{26}$ Bussy D'Ambois: A Tragedie, éd. John Hazel Smith dans The Plays of George Chapman: The Tragedies, op. cit., 1987. 
hope of my revival see me » $(T B$, v.iv.246-47) et plus loin, « but cursèd man / Shall never more renew his vanished face» (TB, v.iv.252-53). Ces vers font suite à ceux dans lesquels Byron constate la dislocation du monde :

Wretched world,

Consisting most of parts that fly each other,

A firmness breeding all inconstancy,

A bond of disjunction; like a man

Long buried is a man that long hath lived ;

Touch him, he falls to ashes [...].

(TB, v.iv.62-67)

Byron s'avoue donc vaincu et offre une figure qui est tout au rebours du phœnix dont parle John Donne dans The First Anniversary: An Anatomy of the World:

'Tis all in peeces, all coherence gone;

All just supply, and all Relation:

Prince, Subject, Father, Sonne, are things forgot,

For every man alone thinkes he hath got

To be a Phoenix, and that then can bee

None of that kinde, of which he is, but hee.

$$
\text { (v. 213-18) } 27
$$

Lui qui imaginait un monument à sa gloire de la taille d'une montagne, demande à présent: «On what region / Must this declining prominent ${ }^{28}$ pour his load? » (v.iv.18-19) pour savoir comment placer son corps par rapport au billot. La réduction de l'épique au trivial est ici frappante et illustre parfaitement le sort de ce « héros inachevé ».

Cette réduction prend, dans ce diptyque, la forme particulière d'une dissolution de l'héroïsme. Dans aucune autre pièce de son œuvre, Chapman n'a autant utilisé les images aquatiques pour figurer la versatilité toute baroque d'un monde instable. Dès le prologue, comme on l'a vu plus haut, on sait que le personnage principal sera soumis aux flux et reflux de la fortune. Or, quel meilleur moyen de signifier le caractère insaisissable de la mémoire que l'eau, sur laquelle nulle empreinte ne peut être laissée, contrairement au bronze des

27 John Donne, Selected Poems, éd. Ilona Bell, Londres, Penguin Classics, 2006, p. 162. 28 Voir $O E D$ sur le sens de «prominent » en tant que substantif : «A prominent person » (B.1.a), avec comme exemple ce passage de The Tragedy of Byron ou «A protruding or projecting part; a prominence. Obs. rare.", avec comme exemple Chapman, l'Iliade d'Homère, chants XI et XII. 
statuaires ? Ainsi, Chapman oppose des images contradictoires quand il s'agit de poser son personnage. Savoy évoque la mer quand il décrit le siège de Dreux : « this duke [...] seconds his father, / Beats through and through the enemy's greatest strength, / And breaks the rest like billows 'gainst a rock » $(C B$, II.ii.129-31). Ici, Byron est le rocher sur lequel les rouleaux se brisent. À la fin, c'est lui que Chapman associe aux rouleaux, en lui faisant déclarer: "I'll break my blood's high billows 'gainst my stars» (TB, v.iv.20). En relatant la bataille de Fontaine-Française, Savoy utilise à nouveau des images aquatiques : « [Byron] beat him [Mayenne] into rout, / And as a little brook being overrun / With a black torrent, that bears all things down / His fury overtakes [...] » ( $C B$, II.ii.187-90). Le torrent, c'est bien entendu Byron, tandis que Mayenne - le gros Mayenne ! - est le ruisseau. C'est par le jeu de ces métaphores que Chapman enclenche le processus de réduction héroïque de Byron. Ainsi, dans la dernière scène de The Conspiracy of Byron, Henry ramène à la raison un Byron hors de lui à qui il finit par pardonner. Dans son discours, on trouve ce passage :

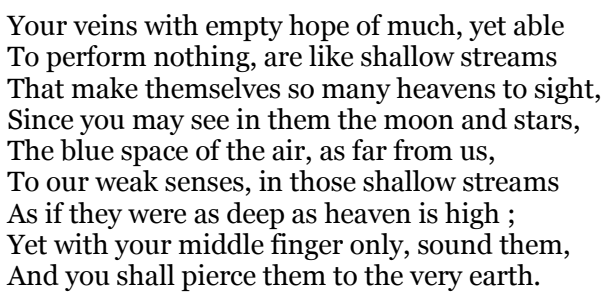

Ici, non seulement le torrent furieux qui emporte tout sur son passage est devenu un petit cours d'eau peu profond, mais en plus ce cours d'eau n'est qu'un miroir aux alouettes, un piège où un Byron narcissique risque de se perdre en oubliant le sens des proportions et de la réalité.

Enfin, on mentionnera la comparaison accablante que le roi utilise à la fin de la scène où Savoy fait l'éloge hyperbolique de Byron. Le roi, soucieux de remettre les choses à leur juste place, rappelle au duc la présence de Lord Norris et du Colonel Williams, qu'il n'hésite pas à comparer à son maréchal. En évoquant ce dernier, il dit : 
And as in open vessels filled with water

And on men's shoulders borne, they put treen cups 29

To keep the wild and slippery element

From washing over, follow all his sways

And tickle aptness to exceed his bounds

And at the brim contain him, so this knight

Swum in Byron, and held him but to right.

Cette métaphore est, pourrait-on dire, programmatique : Henry explique à Savoy que Byron est un «élément fougueux et glissant » (v.234) qu'il faut absolument contenir, quand bien même il faudrait le mettre dans une bassine! Quoi de plus trivial que cette comparaison pour celui qui ne se pense qu'à l'échelle des éléments de la nature et de l'univers. Le but du roi est donc bien de réduire Byron du statut de héros au statut de sujet, voire de sujet mort si nécessaire.

La victoire d'Henry semble totale: une seule mémoire triomphe, le passé de Byron est éliminé au prix d'un oubli forcé et il ne reste que l'histoire du roi Henry, décidément fort proche du Henri IV historique. En effet, si 1598 est la date du Traité de Vervins, c'est aussi celle de l'Édit de Nantes. Or, à quoi sont consacrés les deux premiers articles du texte le plus important du règne d'Henri IV ? À l'oubli, au devoir d'oubli devrait-on plutôt dire :

Article premier :

Que la mémoire de toutes choses passées d'une part et d'autre, depuis le commencement du mois de mars 1585 jusqu'à notre avènement à la couronne, et durant les autres troubles précédents et à l'occasion d'iceux, demeurera éteinte et assoupie, comme de chose non advenue ; et ne sera loisible ni permis à nos procureurs généraux, ni autres personnes quelconques, publiques ni privées, en quelque temps, ni pour quelque occasion que ce soit, en faire mention, procès ou poursuite en aucune cour et juridiction que ce soit.

Article 2 :

Défendons à tous nos sujets de quelque état et qualité qu'ils soient d'en renouveler la mémoire, s'attaquer, injurier ni provoquer l'un l'autre par reproche de ce qui s'est passé, pour quelque cause et prétexte que ce soit, en disputer, contester, quereller ni s'outrager ou s'offenser de fait ou de parole ; mais se contenir et vivre paisiblement ensemble comme frères, amis et concitoyens, sur peine aux contrevenants d'être punis

\footnotetext{
${ }^{29}$ Ce sont des coupes en bois.
} 
comme infracteurs de paix et perturbateurs du repos public ${ }^{30}$. [c'est moi qui souligne]

Cette injonction d'oubli (qui s'applique, entre parenthèses, à la fin du règne de son prédécesseur et doit être distinguée de la damnatio memoriae à laquelle il voue Byron) résonne admirablement avec le processus que Chapman décrit dans sa double pièce : son personnage, à l'image du vrai roi, sent bien quelles conséquences mortifères peuvent avoir une mémoire mal canalisée et un ressassement douloureux.

Ainsi, loin d'être l'«ars memoriae » dont parle Paul Ricœur, qui présente l'ars memoriae comme « un déni outrancier de l'oubli », l'histoire se fait dans les deux cas (réalité et fiction) contre la mémoire, ou, plus exactement, elle se fonde sur la négation autoritaire du passé (« comme de chose non advenue »). On peut affirmer ainsi que Henry met en œuvre un art de l'oubli, qui serait un « déni outrancier de la mémoire ${ }^{31} »$.

Gilles BERTHEAU

Université François-Rabelais, Tours

\footnotetext{
30 Édit de Nantes, cité par Bernard Cottret dans 1598 L'Édit de Nantes : pour en finir avec les guerres de religion, Paris, Perrin, 1997, annexe III, p. 363.

${ }^{31}$ Voir Paul Ricoeur, La Mémoire, l'histoire, l'oubli, op. cit., p. 79 : il présente l'ars memoriae comme « un déni outrancier de l'oubli ».
} 